\title{
Stereochemical Assignment of the Fungal Metabolites Pestalotiopsones D and E through Enantiopure Synthesis
}

\author{
Andrew Michael Beekman and Russell Allan Barrow* \\ Research School of Chemistry, The Australian National University, Canberra, ACT, 0200, Australia
}

Supporting Information

ABSTRACT: The pestalotiopsones are fungal metabolites isolated from an endophytic fungus Pestalotiopsis sp. found in the mangrove Rhizophora mucronata, used in traditional Chinese medicine to treat symptoms of dysentery. The absolute configurations of pestalotiopsones $\mathrm{D}$ (4) and $\mathrm{E}$ (5) were elucidated through total synthesis of both the $R$ and $S$ enantiomers, allowing for the assignment of the stereochemistry of the natural compounds as the (+)-S enantiomers. The key steps include homologation of a substituted benzoic acid to the appropriate phenylacetate derivative using Birch reductive alkylation, an oxa-Michael cyclization induced by microwave irradiation to form the chromanone substructure, and an IBXmediated dehydrogenation yielding the chromone skeleton. Assessment of the synthetic compounds against clinical pathogens was performed.
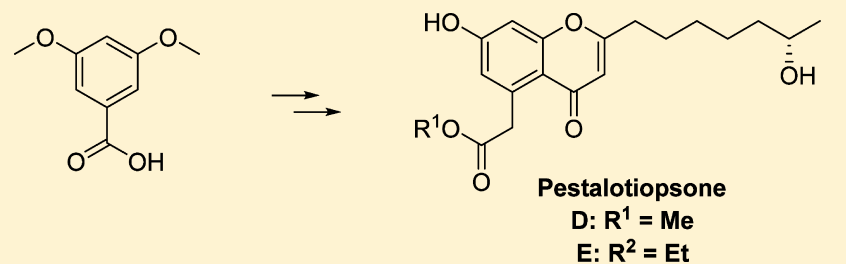

$D: R^{1}=M e$

$E: R^{2}=E t$
T $\mathrm{n}$ the search for new pharmaceuticals ethnopharmacology is a resource that has overwhelming potential. With a large array of prospective structures for medicinal testing the information provided by ethnopharmacology gives research a significant head start. The compounds isolated from traditional medicines often display the traits of proven efficacy and low toxicity, two significant hurdles that face a new drug candidate.

The role that mushrooms play in ethnopharmacology is well documented in traditional Chinese and Ayurvedic medicine, ${ }^{1}$ and the biodiversity of fungi has resulted in a plethora of bioactive molecules with pharmaceutical potential. Additionally, studies have suggested that the activity attributed to some traditional medicinal plants is in fact due to endophytic fungi. ${ }^{2}$

In 2009, Proksch and co-workers isolated a number of chromone derivatives from an endophytic fungal species of the genus Pestalotiopsis cultured from the mangrove Rhizophora mucronata, used as a traditional medicinal to treat symptoms of dysentery, which they named pestalotiopsones A-F (1-6) (Figure 1). ${ }^{3}$ Chromones have been shown to possess a wide range of bioactivity, ${ }^{4}$ and the pestalotiopsones have been shown to exhibit cytotoxic and antibiotic activity. ${ }^{3,5}$ While pestalotiopsones D (4) and E (5) were isolated as optically active compounds $\left([\alpha]_{\mathrm{D}}+16\right.$ and +37 , respectively), the absolute configuration of the compounds was not determined.

The syntheses of pestalotiopsones A, B, C, and F were reported by us recently. ${ }^{5}$ We report here the first synthesis of pestalotiopsones $\mathrm{D}$ and $\mathrm{E}$ as well as ent-D and ent-E, leading to the determination of the absolute configuration of the natural products.

\section{RESULTS AND DISCUSSION}

Pestalotiopsones D (4) and E (5) differ from pestalotiopsones A (1) and B (2) by the existence of a hydroxy group at position 
Scheme 1. Retrosynthesis of Pestalotiopsone D (4)

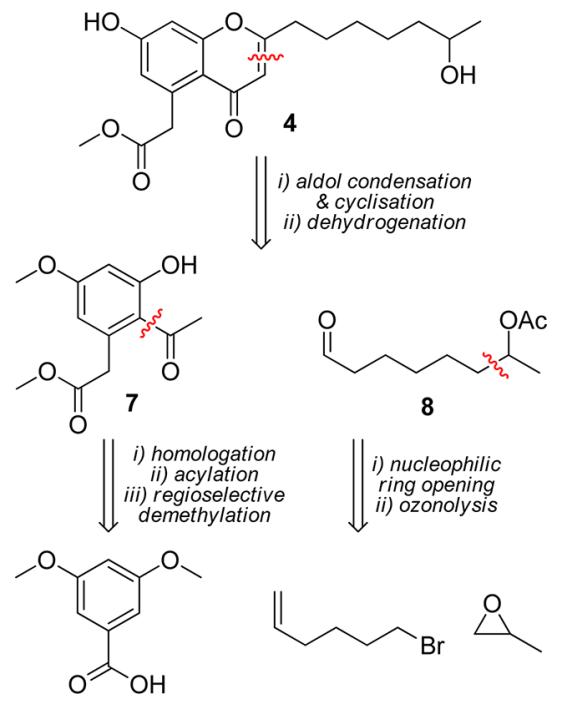

withstand the microwave conditions as well as chromanone oxidation, and so the acetate protecting group was chosen for this task. As such, it was envisaged that aldehyde 8 could be afforded from the ring-opening of enantiopure propylene oxide by the Grignard reagent prepared from 6-bromohex-1-ene, followed by acetate protection and subsequent ozonolysis.

Synthetic work began with the preparation of alcohol $(S)-9$ from enantiopure $(S)$-propylene oxide (Scheme 2), with yields

Scheme 2. Synthesis of Aldehyde (S)-8

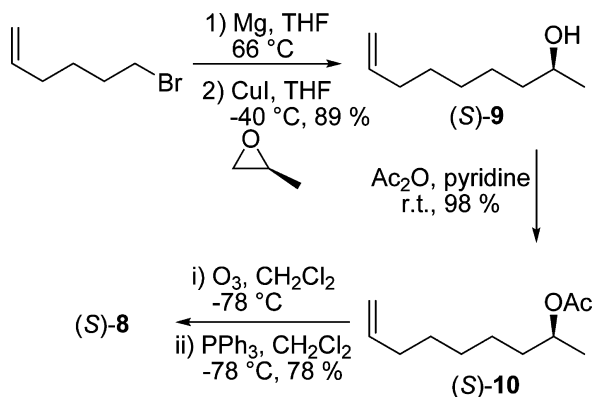

of the desired alcohol significantly increased by addition of cuprous iodide to the preformed Grignard reagent. Protection using pyridine and acetic anhydride yielded acetoxynonene $(S)$ 10 in excellent yield. Conversion to the aldehyde $(S)-8$ was achieved using ozonolysis conditions with reduction of the secondary ozonide being achieved with triphenylphosphine.

With aldehyde $(S)-\mathbf{8}$ in hand the one-pot microwavemediated aldol condensation, dehydration, and oxa-Michael cyclization of the resulting enone could be undertaken (Scheme 3 ). Addition of acetophenone 7 , synthesized as described by us previously, ${ }^{5}$ to aldehyde $(S)-8$ and diisopropylamine in methanol heated to $140{ }^{\circ} \mathrm{C}$ for $60 \mathrm{~min}$ yielded chromanone $\left(6^{\prime \prime} S\right)-11$ in good yield. Despite $\left(6^{\prime \prime} S\right)-11$ being a diastereomeric mixture, both the ${ }^{1} \mathrm{H}$ and ${ }^{13} \mathrm{C}$ NMR data presented as a single compound. Dehydrogenation to chromone $(S)-12$ was achieved with IBX, $^{6}$ and subsequent deprotection with aluminum chloride in refluxing toluene gave $(S)-4$ in excellent yield.

The spectroscopic data of $(S)-4$ corresponded closely to that of the natural 4$)^{3}$ while the specific rotation, $[\alpha]^{20}{ }_{D}+12.6(c$
0.93, $\mathrm{MeOH}$ ), was identical in sign and of comparable magnitude to that of natural 4 isolated by Proksch et al., $[\alpha]^{20}{ }_{\mathrm{D}}+16(c 0.02, \mathrm{MeOH})$. Additionally, the enantiomer $(R)$ 4 synthesized from $(R)$-propylene oxide using the same synthetic procedure returned a specific rotation of $[\alpha]_{D}^{20}$ -10.8 (c $0.81, \mathrm{MeOH})$, opposite in sign of that of the natural 4. Synthesis of $(S)-\mathbf{4}$ and $(R)-4$ has led to the determination of the absolute configuration of pestalotiopsone $\mathrm{D}$ as $S$.

Subsequently, both (S)-4 and (R)-4 were subjected to transesterification conditions with sulfuric acid in ethanol to yield $(S)-5$ and $(R)-5$ in excellent yield (Scheme 3 ). The spectroscopic data matched those of the natural 5 , while the specific rotation of $(S)-5,[\alpha]_{D}^{20}+14.1(c 0.73, \mathrm{MeOH})$, was again identical in sign to that of natural $5,[\alpha]^{20}{ }_{D}+37(c 0.02$, $\mathrm{MeOH}$ ), leading to the assignment of the absolute configuration of pestalotiopsone $\mathrm{E}$ as $S$ also. The difference in magnitude in the specific rotation values between the synthetic $(S)-5$ and the natural $\mathbf{5}$ may be attributed to the small amount of product isolated from the natural source $(0.26 \mathrm{mg}) .^{3}$

The synthetic pestalotiopsones $((S)-4,(S)-5)$ as well as their enantiomers $((R)-4,(R)-5)$, along with a number of synthetic intermediates $\left(\left(6^{\prime \prime} S\right)-11,\left(6^{\prime \prime} R\right)-11,(S)-12,(R)-12\right)$, were tested against clinical strains of Staphylococcus epidermidis, Escherichia coli, Pseudomonas aeruginosa, and Mycobacterium smegmatis. While active, the MIC values for the evaluated compounds against these strains were all greater than $500 \mu \mathrm{g} /$ $\mathrm{mL}$. The $\mathrm{IC}_{50}$ values for the natural enantiomers and their precursors are displayed in Table 1 . While both enantiomers were tested, there was not a significant difference in activity between them, and as such only the $\mathrm{IC}_{50}$ values for the natural isomers are presented. Kanamycin was used in the assay as a positive control and for comparison. Pestalotiopsones D (4) and $\mathrm{E}$ (5) show comparable activity when compared to pestalotiopsones A, B, C, and F $(1-3,6),{ }^{5}$ but possess weak activity when compared to the clinical antibiotic kanamycin.

In conclusion, the first syntheses of pestalotiopsones $\mathrm{D}$ and $\mathrm{E}$ were achieved in seven and eight linear steps, respectively, from commercially available 3,5-dimethoxybenzoic acid, in addition to ent-pestalotiopsone $\mathrm{D}$ and ent-E, allowing for the structural elucidation of natural pestalotiopsones $\mathrm{D}$ and $\mathrm{E}$ as the $S$ enantiomers. Biological testing of the synthetic compounds showed they possessed weak antimicrobial activity against a panel of human pathogens.

\section{EXPERIMENTAL SECTION}

General Experimental Procedures. Reagents were purchased from commercial suppliers and used without further purification unless otherwise stated. All solvents were dried and distilled immediately prior to use or stored as appropriate. Ethereal solvents $\mathrm{Et}_{2} \mathrm{O}$ and THF were refluxed over sodium and benzophenone. Toluene was refluxed over sodium, $\mathrm{CH}_{2} \mathrm{Cl}_{2}$ was refluxed with and distilled from $\mathrm{CaH}_{2}$, and acetone was refluxed with and distilled from $\mathrm{Na}_{2} \mathrm{SO}_{4}$. Petroleum ether used is the $60-80{ }^{\circ} \mathrm{C}$ fraction. Optical data were measured on a Perkin-Elmer 341 polarimeter. Concentrations are expressed as g/100 $\mathrm{mL}$, measured at the sodium $\mathrm{D}$-line $(589 \mathrm{~nm})$ at $20{ }^{\circ} \mathrm{C}$ in the solvent indicated. UV spectra were measured on a Cary $4 \mathrm{G} \mathrm{UV-visible}$ spectrophotometer using a $1 \mathrm{~cm}$ solution cell in the solvent indicated. Spectra maxima are recorded in $\mathrm{nm}(\log \varepsilon)$. IR spectra were recorded on a Bruker Alpha-P ATR spectrophotometer as neat solid or film. Characteristic peaks are recorded in wavenumbers. ${ }^{1} \mathrm{H}$ and ${ }^{13} \mathrm{C}$ NMR spectra were recorded at $298 \mathrm{~K}$, at $300 \mathrm{MHz}$ or $400 \mathrm{MHz}$ and 75, 100 or $125 \mathrm{MHz}$, on an Inova 300, Varian Mercury 300, Varian MR-400, or Inova 500 instrument. Chemical shifts are reported in $\mathrm{ppm}(\delta) .{ }^{1} \mathrm{H}$ NMR spectra are referenced to the resonance from residual $\mathrm{CHCl}_{3}$ at $7.26 \mathrm{ppm}$ or the central peak in the resonance from residual 
Scheme 3. Synthesis of (S)-Pestalotiopsone D (4) and (S)-Pestalotiopsone E (5)

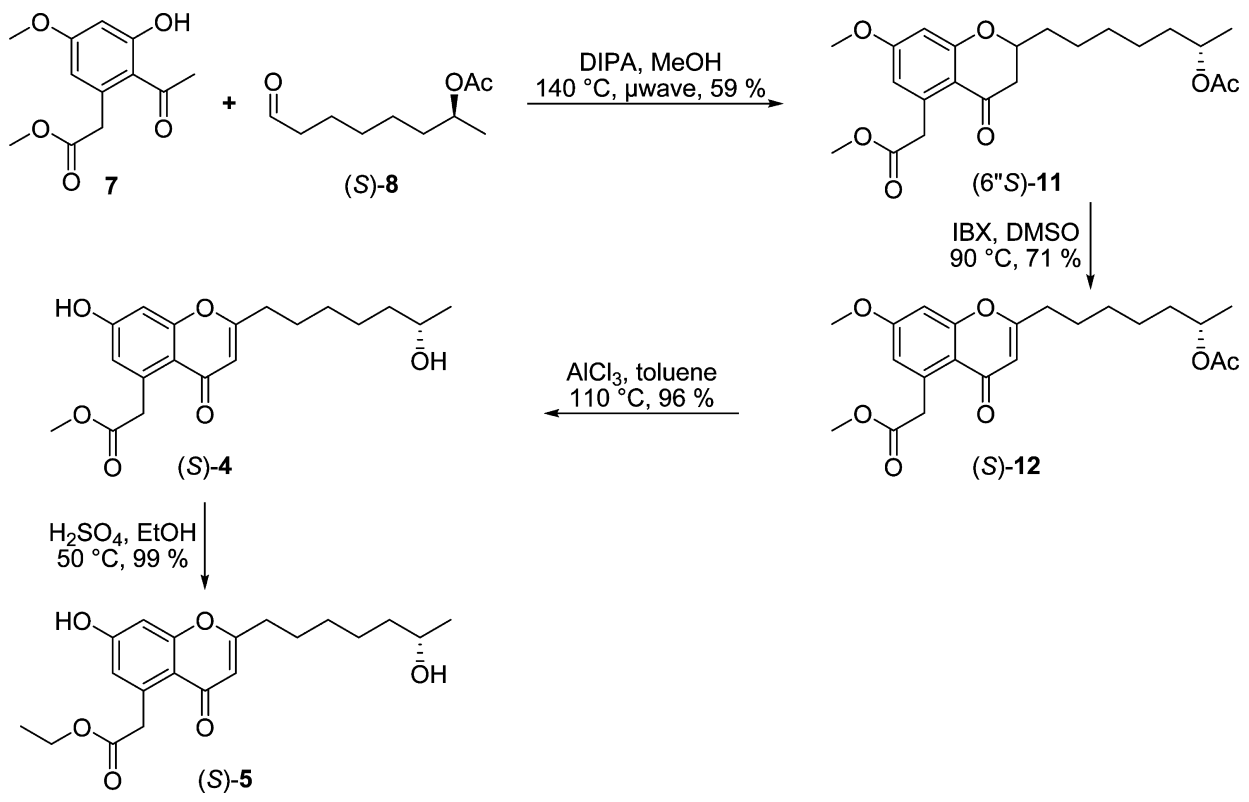

Table 1. Antibiotic Activity of $(S)-4,(S)-5,\left(6^{\prime \prime} S\right)-11$, and $(S)-12$ Showing $50 \%$ Inhibition of Selected Strains

\begin{tabular}{lrrrcr} 
& \multicolumn{5}{c}{$\mathrm{IC}_{50}(\mu \mathrm{g} / \mathrm{mL})$} \\
\cline { 2 - 6 } \multicolumn{1}{c}{ strain $^{a}$} & $\operatorname{kan}^{b}$ & $(S)-4$ & $(S)-5$ & $\left(6^{\prime \prime} S\right)-11$ & $(S)-12$ \\
S. epidermidis & 90 & $>500$ & $>500$ & $>500$ & $>500$ \\
E. coli & $<1$ & $>500$ & $>500$ & $>500$ & $>500$ \\
$P$. aeruginosa & 209 & $>500$ & 440 & 445 & 465 \\
M. smegmatis & $<1$ & $>500$ & 484 & 492 & $>500$
\end{tabular}

${ }^{a}$ Clinical strains obtained from The Canberra Hospital. ${ }^{b}$ Kanamycin.

$\mathrm{CHD}_{2} \mathrm{COCD}_{3}$ at $2.05 \mathrm{ppm} .{ }^{13} \mathrm{C}$ NMR spectra are referenced to the central peak in the signal from $\mathrm{CDCl}_{3}$ at $77.0 \mathrm{ppm}$ or the central peak in the resonance from $\left(\mathrm{CD}_{3}\right)_{2} \mathrm{CO}$ at $29.8 \mathrm{ppm}$. The appearance and multiplicities of ${ }^{1} \mathrm{H}$ resonances are expressed by the abbreviations $\mathrm{s}$ (singlet), $\mathrm{d}$ (doublet), $\mathrm{t}$ (triplet), $\mathrm{q}$ (quartet), and $\mathrm{m}$ (multiplet) and combinations thereof for more highly coupled systems. ${ }^{13} \mathrm{C}$ NMR experiments were run as proton-decoupled spectra. ${ }^{1} \mathrm{H}$ signals and ${ }^{13} \mathrm{C}$ signals where appropriate are described by chemical shift $\delta$ (integration, multiplicity, $\mid \mathrm{Jl}(\mathrm{Hz})$, assignment). When ambiguous, assignments were made based on appropriate 2D NMR data, including HSQC, HMBC, and NOESY. EIMS and HREIMS were recorded on a VG autospec mass spectrometer or Waters Autospec Premier mass spectrometer, operating at $70 \mathrm{eV}$. ESIMS and HRESIMS were recorded on a Bruker Apex 3. Positive ionization was detected unless otherwise indicated. Mass/charge ratios $(\mathrm{m} / z)$ are reported and relative abundance of the ions as percentage of base peak intensity. Thin layer chromatography (TLC) was run on Merck silica gel $60 \mathrm{~F}_{254}$ aluminum-backed plates and was run in the eluting system described for each plate. Flash chromatography was performed under pressure using silica gel (230-400 mesh Scharlau 60) as solid support and HPLC-grade solvents as eluent. Analytical HPLC was performed using an Agilent 1100 system, utilizing a Phenomenex LUX cellulose-4 $5 \mu \mathrm{m}$ column and an Agilent 1100 series diode array detector for eluate detection. Elution was carried out using prefiltered $(0.45 \mu \mathrm{m}$ nylon membrane filter) ethanol and $n$-hexane in the ratio indicated, at 0.5 $\mathrm{mL} / \mathrm{min}$ with detection at $243 \mathrm{~nm}$. Microwave reactions were carried out using an Explorer CEM Automated Microwave Workstation in 10 $\mathrm{mL}$ reaction vessels at the wattage $(\mathrm{W})$ and pressure stated.

Methyl 2-(2-Acetyl-3-hydroxy-5-methoxyphenyl)acetate (7). To a solution of 3,5-dimethoxybenzoic acid $(1.82 \mathrm{~g}, 10.0 \mathrm{mmol})$ in THF $(20 \mathrm{~mL})$ was condensed $\mathrm{NH}_{3}(50 \mathrm{~mL})$, and lithium $(153 \mathrm{mg}, 21.9$ mmol) was added in portions at $-33^{\circ} \mathrm{C}$. Methyl bromoacetate $(1.14$
$\mathrm{mL}, 1.84 \mathrm{~g}, 12.0 \mathrm{mmol}$ ) was added dropwise, causing the deep blue solution to become a pale yellow color. The $\mathrm{NH}_{3}$ was evaporated under a stream of nitrogen, and the resulting solid residues were dissolved in water and $\mathrm{Et}_{2} \mathrm{O}$. The layers were separated, and the aqueous layer was cooled to $0{ }^{\circ} \mathrm{C}$ and acidified to $\mathrm{pH} 4$ with careful addition of aqueous $1 \mathrm{M} \mathrm{HCl}$. The acidic solution was then extracted with EtOAc, dried with $\mathrm{MgSO}_{4}$, filtered, and concentrated in vacuo to give 3,5-dimethoxy-1-(2-methoxy-2-oxoethyl)cyclohexa-2,5-dienecarboxylic acid as a colorless solid $(93 \%, 2.38 \mathrm{~g}, 9.30 \mathrm{mmol})$. IR (neat): $\nu_{\max } 2982,2651,1703,1649,1695 \mathrm{~cm}^{-1} ;{ }^{1} \mathrm{H}$ NMR $(300 \mathrm{MHz}$, $\left.\mathrm{CDCl}_{3}\right) \delta 4.77(2 \mathrm{H}, \mathrm{s}, 2,6-\mathrm{H}), 3.67\left(3 \mathrm{H}, \mathrm{s}, 2^{\prime}-\mathrm{OCH}_{3}\right), 3.58(6 \mathrm{H}, \mathrm{s}, 3,5-$ $\left.\mathrm{OCH}_{3}\right), 2.7-2.9\left(4 \mathrm{H}, \mathrm{m}, 4-\mathrm{H}_{\mathrm{A}}, 4-\mathrm{H}_{\mathrm{B}}, 1^{\prime}-\mathrm{H}\right) ;{ }^{13} \mathrm{C}$ NMR $(75 \mathrm{MHz}$, $\left.\mathrm{CDCl}_{3}\right) \delta 180.9(\mathrm{COOH}), 171.4\left(2^{\prime}-\mathrm{C}\right), 154.2$ (3,5-C), 93.9 (2,6-C), $54.5\left(3,5-\mathrm{OCH}_{3}\right), 51.6\left(2^{\prime}-\mathrm{OCH}_{3}\right), 47.8(1-\mathrm{C}), 45.6\left(1^{\prime}-\mathrm{C}\right), 31.0(4-$ C); ESIMS $257\left(100,[\mathrm{M}+\mathrm{H}]^{+}\right)$; HRESIMS $m / z 257.1024[\mathrm{M}+\mathrm{H}]^{+}$ (calcd for $\mathrm{C}_{12} \mathrm{H}_{17} \mathrm{O}_{6}, 257.1025$ ).

To a solution of 3,5-dimethoxy-1-(2-methoxy-2-oxoethyl)cyclohexa2,5-dienecarboxylic acid $(1.85 \mathrm{~g}, 7.20 \mathrm{mmol})$ in toluene $(150 \mathrm{~mL})$ was added lead tetraacetate $(4.15 \mathrm{~g}, 9.40 \mathrm{mmol})$. After $40 \mathrm{~min}$ water was added and the mixture was filtered through a pad of silica. The organic phase was separated, washed with saturated aqueous $\mathrm{NaHCO}_{3}$, dried with $\mathrm{MgSO}_{4}$, filtered, and concentrated in vacuo to give an orange oil. Purification was achieved by flash column chromatography on silica with $33 \%$ EtOAc in petroleum ether as the eluent $\left(R_{f}=0.6\right)$ to yield methyl 2-(3,5-dimethoxyphenyl)acetate as a pale yellow oil (91\%, 1.38 g, $6.55 \mathrm{mmol}$ ): IR (neat) $\nu_{\max } 1723,1602 \mathrm{~cm}^{-1}$; ${ }^{1} \mathrm{H}$ NMR $(300 \mathrm{MHz}$, $\left.\mathrm{CDCl}_{3}\right) \delta 6.44\left(2 \mathrm{H}, \mathrm{d},{ }^{4} J=2.3 \mathrm{~Hz}, 2^{\prime}, 6^{\prime}-\mathrm{H}\right), 6.37\left(1 \mathrm{H}, \mathrm{t},{ }^{4} J=2.3 \mathrm{~Hz}\right.$, $\left.4^{\prime}-\mathrm{H}\right), 3.78\left(6 \mathrm{H}, 3^{\prime}, 5^{\prime}-\mathrm{OCH}_{3}\right), 3.69\left(3 \mathrm{H}, \mathrm{s}, 1-\mathrm{OCH}_{3}\right), 3.56(2 \mathrm{H}, \mathrm{s}, 2-$ $\mathrm{H}) ;{ }^{13} \mathrm{C}$ NMR (75 MHz, $\left.\mathrm{CDCl}_{3}\right) \delta 171.8$ (1-C), $160.8\left(3^{\prime}, 5^{\prime}-\mathrm{C}\right), 136.0$ $\left(1^{\prime}-\mathrm{C}\right), 107.3\left(2^{\prime}, 6^{\prime}-\mathrm{C}\right), 99.2\left(4^{\prime}-\mathrm{C}\right), 55.3\left(3^{\prime}, 5^{\prime}-\mathrm{OCH}_{3}\right), 52.1$ (1$\left.\mathrm{OCH}_{3}\right), 41.4(2-\mathrm{C})$; EIMS $210\left(100,[\mathrm{M}]^{+\bullet}\right), 151(95), 121(30), 91$ (20); HREIMS $m / z 210.0890[\mathrm{M}]^{+\bullet}\left(\right.$ calcd for $\left.\mathrm{C}_{11} \mathrm{H}_{14} \mathrm{O}_{4}, 210.0892\right)$.

To a solution of methyl 2-(3,5-dimethoxyphenyl)acetate $(210 \mathrm{mg}$, $1.00 \mathrm{mmol})$ and acetic anhydride $(0.950 \mathrm{~mL}, 1.02 \mathrm{~g}, 10.0 \mathrm{mmol})$ in acetic acid $(20 \mathrm{~mL})$ at $35^{\circ} \mathrm{C}$ was added perchloric acid $(70 \%, 1.44 \mathrm{~mL}$, $10.0 \mathrm{mmol}$ ) dropwise. The solution was stirred for $5 \mathrm{~min}$ and then poured into ice water. $\mathrm{Et}_{2} \mathrm{O}$ and saturated aqueous $\mathrm{NaHCO}_{3}$ were added, and the organic layer was separated. The organic layer was washed with saturated aqueous $\mathrm{NaHCO}_{3}$, dried with $\mathrm{MgSO}_{4}$, filtered, and concentrated in vacuo to give a yellow oil. Purification was achieved by flash column chromatography on silica with $33 \%$ EtOAc in petroleum ether as eluent $\left(R_{f}=0.4\right)$, yielding methyl 2-(2-acetyl-3,5dimethoxyphenyl)acetate as a pale yellow, amorphous solid (89\%, 224 $\mathrm{mg}, 0.890 \mathrm{mmol}$ ): IR (neat) $\nu_{\max } 1745,1686 \mathrm{~cm}^{-1}$; ${ }^{1} \mathrm{H}$ NMR (300 
$\left.\mathrm{MHz}, \mathrm{CDCl}_{3}\right) \delta 6.40\left(1 \mathrm{H}, \mathrm{d},{ }^{4} J=2.2 \mathrm{~Hz}, 6^{\prime}-\mathrm{H}\right), 6.34\left(1 \mathrm{H}, \mathrm{d},{ }^{4} J=2.2\right.$ $\left.\mathrm{Hz}, 4^{\prime}-\mathrm{H}\right), 3.82\left(3 \mathrm{H}, \mathrm{s}, 5^{\prime}-\mathrm{OCH}_{3}\right), 3.80\left(3 \mathrm{H}, \mathrm{s}, 3^{\prime}-\mathrm{OCH}_{3}\right), 3.68(2 \mathrm{H}, \mathrm{s}$, 2-H), $3.66\left(3 \mathrm{H}, \mathrm{s}, 1-\mathrm{OCH}_{3}\right), 2.49\left(3 \mathrm{H}, \mathrm{s}, \mathrm{C}(\mathrm{O}) \mathrm{CH}_{3}\right) ;{ }^{13} \mathrm{C} \mathrm{NMR}(75$ $\left.\mathrm{MHz}, \mathrm{CDCl}_{3}\right) \delta 203.7\left(\mathrm{C}(\mathrm{O}) \mathrm{CH}_{3}\right), 171.7$ (1-C), $161.5\left(5^{\prime}-\mathrm{C}\right), 159.4$ (3'-C), 134.9 (1'-C), $123.6\left(6^{\prime}-\mathrm{C}\right), 108.2\left(2^{\prime}-\mathrm{C}\right), 97.4\left(4^{\prime}-\mathrm{C}\right), 55.6$ (5'$\left.\mathrm{OCH}_{3}\right), 55.3\left(3^{\prime}-\mathrm{OCH}_{3}\right), 51.9\left(1-\mathrm{OCH}_{3}\right), 39.0(2-\mathrm{C}), 32.2(\mathrm{C}(\mathrm{O})$ $\left.\mathrm{CH}_{3}\right)$; EIMS $252\left(55,[\mathrm{M}]^{+\bullet}\right), 237$ (60), 221 (35), 209 (100), 192 (65), 178 (50); HREIMS $\mathrm{m} / z 252.0996[\mathrm{M}]^{+\bullet}\left(\right.$ calcd for $\mathrm{C}_{13} \mathrm{H}_{16} \mathrm{O}_{5}$, 252.0998).

To a solution of methyl 2-(2-acetyl-3,5-dimethoxyphenyl)acetate (913 $\mathrm{mg}, 3.62 \mathrm{mmol})$ in $\mathrm{CH}_{2} \mathrm{Cl}_{2}(15 \mathrm{~mL})$ at $-78{ }^{\circ} \mathrm{C}$ under an atmosphere of argon was added boron trichloride ( $1 \mathrm{M} n$-hexane solution, $5.43 \mathrm{~mL}, 5.43 \mathrm{mmol}$ ) dropwise. The solution was allowed to slowly warm to room temperature and stirred for $16 \mathrm{~h}$, at which time ice $(5 \mathrm{~g})$ was added. The solution was partitioned between water and $\mathrm{CH}_{2} \mathrm{Cl}_{2}$, and the organic layer separated, dried with $\mathrm{MgSO}_{4}$, filtered, and concentrated under reduced pressure to give the title compound as a colorless solid $(93 \%, 801 \mathrm{mg}, 3.37 \mathrm{mmol})$ : IR (neat) $\nu_{\max } 3268$, 1701, $1598 \mathrm{~cm}^{-1}$; ${ }^{1} \mathrm{H}$ NMR $\left(300 \mathrm{MHz}, \mathrm{CDCl}_{3}\right) \delta 13.06\left(1 \mathrm{H}, \mathrm{s}, 3^{\prime}-\right.$ $\mathrm{OH}), 6.38\left(1 \mathrm{H}, \mathrm{d},{ }^{4} J=1.7 \mathrm{~Hz}, 6^{\prime}-\mathrm{H}\right), 6.32\left(1 \mathrm{H}, \mathrm{d},{ }^{4} J=1.7 \mathrm{~Hz}, 4^{\prime}-\mathrm{H}\right)$, $3.90(2 \mathrm{H}, \mathrm{s}, 2-\mathrm{H}), 3.81\left(3 \mathrm{H}, \mathrm{s}, 5^{\prime}-\mathrm{OCH}_{3}\right), 3.72\left(3 \mathrm{H}, \mathrm{s}, 1-\mathrm{OCH}_{3}\right), 2.60$ $\left(3 \mathrm{H}, \mathrm{s}, \mathrm{C}(\mathrm{O}) \mathrm{CH}_{3}\right) ;{ }^{13} \mathrm{C} \mathrm{NMR}\left(75 \mathrm{MHz}, \mathrm{CDCl}_{3}\right) \delta 203.3\left(\mathrm{C}(\mathrm{O}) \mathrm{CH}_{3}\right)$, 171.3 (1-C), 166.2 (5'-C), 164.0 (3'-C), 136.7 (1'-C), $115.4\left(2^{\prime}-\mathrm{C}\right)$, $112.8\left(6^{\prime}-\mathrm{C}\right), 100.4\left(4^{\prime}-\mathrm{C}\right), 55.4\left(5^{\prime}-\mathrm{OCH}_{3}\right), 52.4\left(1-\mathrm{OCH}_{3}\right), 41.7(2-$ C), $31.9\left(\mathrm{C}(\mathrm{O}) \mathrm{CH}_{3}\right)$; EIMS $238\left(50,\left[\mathrm{M}^{+\bullet}\right), 195(100), 178(60)\right.$, 164 (50); HREIMS $\mathrm{m} / z 238.0843[\mathrm{M}]^{+\bullet}$ (calcd for $\mathrm{C}_{12} \mathrm{H}_{14} \mathrm{O}_{5}$, 238.0841).

(S)-Non-8-en-2-ol ((S)-9). To a solution of copper iodide (286 mg, $1.5 \mathrm{mmol})$ in THF $(10 \mathrm{~mL})$ at $-40{ }^{\circ} \mathrm{C}$ was added freshly prepared hex-5-en-1-ylmagnesium bromide (from $0.530 \mathrm{~g}, 22.0 \mathrm{mmol}$ of magnesium and $2.68 \mathrm{~mL}, 3.26 \mathrm{~g}, 20.0 \mathrm{mmol}$ of 6-bromohex-1-ene in $20 \mathrm{~mL}$ of THF), and the mixture was stirred for $15 \mathrm{~min}$. (S)-Propylene oxide $(1.40 \mathrm{~mL}, 1.16 \mathrm{~g}, 20.0 \mathrm{mmol})$ was then added dropwise, and the solution was allowed to stir at $-40{ }^{\circ} \mathrm{C}$ for $4 \mathrm{~h}$. Aqueous $\mathrm{NH}_{4} \mathrm{Cl}$ was then added, and the solution extracted with $\mathrm{Et}_{2} \mathrm{O}$. The organic layer was separated, dried with $\mathrm{MgSO}_{4}$, filtered, and concentrated to give the title compound as a colorless oil $(89 \%, 2.53 \mathrm{~g}, 17.8 \mathrm{mmol}):[\alpha]^{20}{ }_{\mathrm{D}}$ +8.2 (c 1.23, MeOH); IR (neat) $\nu_{\max } 3398,3081,1639 \mathrm{~cm}^{-1} ;{ }^{1} \mathrm{H}$ NMR $\left(300 \mathrm{MHz}, \mathrm{CDCl}_{3}\right) \delta 5.81\left(1 \mathrm{H}, \mathrm{ddt},{ }^{3} \mathrm{~J}=16.9,10.3,6.6 \mathrm{~Hz}, 8\right.$ H), 5.0-4.9 (2H, m, 9-H), 3.9-3.7 (1H, m, 2-H), 2.1-2.0 (2H, m, 7$\mathrm{H}), 1.5-1.2(8 \mathrm{H}, \mathrm{m}, 3-6-\mathrm{H}), 1.18\left(3 \mathrm{H}, \mathrm{d},{ }^{3} \mathrm{~J}=6.2 \mathrm{~Hz}, 1-\mathrm{H}\right) ;{ }^{13} \mathrm{C}$ NMR (75 MHz, CDCl $) \delta 139.0$ (8-C), 114.2 (9-C), 68.1 (2-C), 39.3 (3-C), 33.7 (7-C), 29.1, 28.9, 25.6 (4-C), 23.5 (1-C); EIMS 142 (60, $\left.[\mathrm{M}]^{+\bullet}\right), 127(100)$; HREIMS $m / z 142.1356[\mathrm{M}]^{+\bullet}\left(\right.$ calcd for $\mathrm{C}_{9} \mathrm{H}_{18} \mathrm{O}$, $142.1358)$.

(R)-Non-8-en-2-ol ((R)-9). The title compound was synthesized in the same manner as $(S)-9$ from $(R)$-propylene oxide: $[\alpha]^{20}{ }_{D}-8.9(c$ $1.16, \mathrm{MeOH})$. All remaining spectroscopic data were identical to $(S)$ non-8-en-2-ol.

(S)-Non-8-en-2-yl Acetate ((S)-10). To a solution of (S)-9 (2.42 g, $17.0 \mathrm{mmol})$ in pyridine $(3.00 \mathrm{~mL}, 2.95 \mathrm{~g}, 37.2 \mathrm{mmol})$ was added acetic anhydride $(3.00 \mathrm{~mL}, 3.25 \mathrm{~g}, 31.8 \mathrm{mmol})$, and the solution was stirred at room temperature for $3 \mathrm{~h}$. Aqueous $\mathrm{NH}_{4} \mathrm{Cl}$ was added, and the solution was extracted with $\mathrm{Et}_{2} \mathrm{O}$. The organic layer was then washed with aqueous $\mathrm{NH}_{4} \mathrm{Cl}$ and saturated aqueous $\mathrm{NaHCO}_{3}$, dried with $\mathrm{MgSO}_{4}$, filtered, and concentrated to give a colorless oil $(98 \%, 3.07 \mathrm{~g}$, $16.7 \mathrm{mmol}):[\alpha]^{20}{ }_{\mathrm{D}}+8.3$ (c 10.6, $\left.\mathrm{MeOH}\right)$; IR (neat) $\nu_{\max } 1742,1235$ $\mathrm{cm}^{-1}$; ${ }^{1} \mathrm{H}$ NMR $\left(400 \mathrm{MHz}, \mathrm{CDCl}_{3}\right) \delta 5.80\left(1 \mathrm{H}, \mathrm{ddt},{ }^{3} \mathrm{~J}=17.0,10.2\right.$, $6.7 \mathrm{~Hz}, 8-\mathrm{H}), 5.1-4.8(3 \mathrm{H}, \mathrm{m}, 2,9-\mathrm{H}), 2.1-2.0(2 \mathrm{H}, \mathrm{m}, 7-\mathrm{H}), 2.02$ $\left(3 \mathrm{H}, \mathrm{s}, \mathrm{C}(\mathrm{O}) \mathrm{CH}_{3}\right), 1.8-1.2(8 \mathrm{H}, \mathrm{m}, 3-6-\mathrm{H}), 1.20\left(3 \mathrm{H}, \mathrm{d},{ }^{3} \mathrm{~J}=6.3 \mathrm{~Hz}\right.$, 1-H); ${ }^{13} \mathrm{C}$ NMR $\left(100 \mathrm{MHz}, \mathrm{CDCl}_{3}\right) \delta 170.8\left(\mathrm{C}(\mathrm{O}) \mathrm{CH}_{3}\right), 140.1(8-$ C), 114.3 (9-C), 71.0 (2-C), 35.9 (3-C), 33.7 (7-C), 28.9, 28.8, 25.2 (4-C), $21.4\left(\mathrm{C}(\mathrm{O}) \mathrm{CH}_{3}\right), 20.0$ (1-C); EIMS $184\left(40,[\mathrm{M}]^{+\bullet}\right)$, 137(100); HREIMS $\mathrm{m} / z$ 184.1466 $[\mathrm{M}]^{\bullet}$ (calcd for $\mathrm{C}_{11} \mathrm{H}_{20} \mathrm{O}_{2}$, 184.1463).

(R)-Non-8-en-2-yl Acetate ((R)-10). The title compound was synthesized in the same manner as $(S)-10$ from $(R)-9$ : $[\alpha]^{20}{ }_{D}-7.8$ (c 10.2, MeOH). All remaining spectroscopic data were identical to those of $(S)$-non-8-en-2-yl acetate.
(S)-8-Oxooctan-2-yl Acetate ((S)-8). (S)-10 (2.76 g, $15.0 \mathrm{mmol})$ was dissolved in $\mathrm{CH}_{2} \mathrm{Cl}_{2}(40 \mathrm{~mL})$ and cooled to $-78{ }^{\circ} \mathrm{C}$. Ozone was then bubbled through the solution until a blue color persisted, at which time oxygen was bubbled through the solution for $15 \mathrm{~min}$. Triphenylphosphine $(7.87 \mathrm{~g}, 30.0 \mathrm{mmol})$ was then added to the solution, and the reaction mixture was allowed to slowly warm to room temperature. The solvent was removed in vacuo, and the crude product was purified via flash column chromatography on silica using $10 \%$ EtOAc in petroleum ether as eluent $\left(R_{f}=0.2\right)$, yielding a colorless oil $(78 \%, 2.8 \mathrm{~g}, 11.7 \mathrm{mmol}):[\alpha]_{\mathrm{D}}^{20}+2.0(c 10.3, \mathrm{MeOH})$; IR (neat) $\nu_{\max }$ 2981, 2733, 1750, $1728 \mathrm{~cm}^{-1}$; ${ }^{1} \mathrm{H}$ NMR $\left(300 \mathrm{MHz}, \mathrm{CDCl}_{3}\right) \delta 9.76$ $\left(1 \mathrm{H}, \mathrm{t},{ }^{3} \mathrm{~J}=1.8 \mathrm{~Hz}, 8-\mathrm{H}\right), 5.00-4.75(1 \mathrm{H}, \mathrm{m}, 2-\mathrm{H}), 2.42\left(2 \mathrm{H}, \mathrm{dt},{ }^{3} J=\right.$ $1.8,7.3 \mathrm{~Hz}, 7-\mathrm{H}), 2.02\left(3 \mathrm{H}, \mathrm{s}, \mathrm{C}(\mathrm{O}) \mathrm{CH}_{3}\right), 1.7-1.2(8 \mathrm{H}, \mathrm{m}, 3-6-\mathrm{H})$, $1.19\left(3 \mathrm{H}, \mathrm{d},{ }^{3} \mathrm{~J}=6.2 \mathrm{~Hz}, 1-\mathrm{H}\right) ;{ }^{13} \mathrm{C} \mathrm{NMR}\left(100 \mathrm{MHz}, \mathrm{CDCl}_{3}\right) \delta 202.4$ (8-C), $170.6\left(\mathrm{C}(\mathrm{O}) \mathrm{CH}_{3}\right), 70.7$ (2-C), 43.6 (7-C), 35.5 (3-C), 28.7, 25.0, 21.7, $21.2\left(\mathrm{C}(\mathrm{O}) \mathrm{CH}_{3}\right), 19.8$ (1-C); ESIMS 209 (100, $[\mathrm{M}+$ $\mathrm{Na}]^{+}$); HRESIMS $m / z 209.1155[\mathrm{M}+\mathrm{Na}]^{+}\left(\right.$calcd for $\mathrm{C}_{10} \mathrm{H}_{18} \mathrm{O}_{3}{ }^{23} \mathrm{Na}$, 209.1154).

(R)-8-Oxooctan-2-yl Acetate ((R)-8). The title compound was synthesized in the same manner as $(S)-8$ from $(R)-10:[\alpha]^{20}{ }_{D}-3.1(c$ $10.6, \mathrm{MeOH})$. All remaining spectroscopic data were identical to those of $(S)$-8-oxooctan-2-yl acetate.

$\left(2^{\prime} R, 6^{\prime \prime} S\right)$ and (2'S,6"S)-Methyl 2-(2-(6-Acetoxyheptyl)-7-methoxy-4-oxochroman-5-yl)acetate ((6"S)-11). Diisopropylamine $(140.0 \mu \mathrm{L}, 101.0 \mathrm{mg}, 1.00 \mathrm{mmol})$ and $(S)-8(186.0 \mathrm{mg}, 1.00 \mathrm{mmol})$ were added to a solution of $7(238 \mathrm{mg}, 1.00 \mathrm{mmol})$ in anhydrous methanol $(2 \mathrm{~mL})$, and the mixture was heated to $140{ }^{\circ} \mathrm{C}$ in a microwave reactor $(200 \mathrm{~W}, 200 \mathrm{psi})$ and stirred for $1 \mathrm{~h}$. The resulting dark red solution was diluted with $\mathrm{CH}_{2} \mathrm{Cl}_{2}$ and was subsequently washed with saturated aqueous $\mathrm{NaHCO}_{3}$, water, and brine. The organic layer was then dried with $\mathrm{MgSO}_{4}$, filtered, and concentrated in vacuo to yield an orange oil. Purification was achieved via flash column chromatography on silica with $33 \%$ EtOAc in petroleum ether as eluent $\left(R_{f}=0.3\right)$ to yield the title compounds as an amorphous solid (59\%, $238 \mathrm{mg}, 0.587 \mathrm{mmol}):[\alpha]_{\mathrm{D}}^{20}+26.7$ (c 4.20, MeOH); IR (neat) $\nu_{\max } 2916,1742,1730,1600 \mathrm{~cm}^{-1} ;{ }^{1} \mathrm{H}$ NMR $\left(300 \mathrm{MHz}, \mathrm{CDCl}_{3}\right) \delta$ $6.39\left(1 \mathrm{H}, \mathrm{d},{ }^{4} J=2.3 \mathrm{~Hz}, 6^{\prime}-\mathrm{H}\right), 6.34\left(1 \mathrm{H}, \mathrm{d},{ }^{4} J=2.3 \mathrm{~Hz}, 8^{\prime}-\mathrm{H}\right), 4.88$ $\left(1 \mathrm{H}, \mathrm{tq},{ }^{3} \mathrm{~J}=6.2,6.2 \mathrm{~Hz}, 6^{\prime \prime}-\mathrm{H}\right) 4.39\left(1 \mathrm{H}, \mathrm{dddd},{ }^{3} \mathrm{~J}=4.0,4.8,7.3,11.7\right.$ $\left.\mathrm{Hz}, 2^{\prime}-\mathrm{H}\right), 3.96\left(1 \mathrm{H}, \mathrm{d},{ }^{2} J=16.7 \mathrm{~Hz}, 2-\mathrm{H}_{\mathrm{A}}\right), 3.88\left(1 \mathrm{H}, \mathrm{d},{ }^{2} J=16.7 \mathrm{~Hz}\right.$, $\left.2-\mathrm{H}_{\mathrm{B}}\right), 3.81\left(3 \mathrm{H}, \mathrm{s}, 7^{\prime}-\mathrm{OCH}_{3}\right), 3.70\left(3 \mathrm{H}, \mathrm{s}, 1-\mathrm{OCH}_{3}\right), 2.62\left(1 \mathrm{H}, \mathrm{dd},{ }^{2} \mathrm{~J}\right.$ $\left.=12.5 \mathrm{~Hz},{ }^{3} \mathrm{~J}=11.7 \mathrm{~Hz}, 3^{\prime}-\mathrm{H}_{\mathrm{A}}\right), 2.54\left(1 \mathrm{H}, \mathrm{dd},{ }^{2} J=12.5 \mathrm{~Hz},{ }^{3} J=4.0\right.$ $\left.\mathrm{Hz}, 3^{\prime}-\mathrm{H}_{\mathrm{B}}\right), 2.02\left(3 \mathrm{H}, \mathrm{s}, \mathrm{C}(\mathrm{O}) \mathrm{CH}_{3}\right), 1.9-1.2\left(10 \mathrm{H}, \mathrm{m}, 1^{\prime \prime}-5^{\prime \prime}-\mathrm{H}\right)$, $1.20\left(3 \mathrm{H}, \mathrm{d},{ }^{3} \mathrm{~J}=6.2 \mathrm{~Hz}, 7^{\prime \prime}-\mathrm{H}\right) ;{ }^{13} \mathrm{C}$ NMR $\left(75 \mathrm{MHz}, \mathrm{CDCl}_{3}\right) \delta 192.4$ (4'-C), $171.8(1-\mathrm{C}), 170.9\left(\mathrm{C}(\mathrm{O}) \mathrm{CH}_{3}\right), 165.1\left(7^{\prime}-\mathrm{C}\right), 164.5\left(8^{\prime} \mathrm{a}-\mathrm{C}\right)$, 138.4 (5'-C), 113.9 (6'-C), 113.2 ( $\left.4^{\prime} \mathrm{a}-\mathrm{C}\right), 100.3\left(8^{\prime}-\mathrm{C}\right), 77.4\left(2^{\prime}-\mathrm{C}\right)$, 70.9 (6"-C), $55.4\left(7^{\prime}-\mathrm{OCH}_{3}\right), 51.8\left(1-\mathrm{OCH}_{3}\right), 43.5(2-\mathrm{H}), 41.2\left(3^{\prime}-\right.$ $\mathrm{H}), 35.8\left(5^{\prime \prime}-\mathrm{C}\right) 34.7,29.2$, 25.2, 24.7, $21.4\left(\mathrm{C}(\mathrm{O}) \mathrm{CH}_{3}\right)$, 19.9; EIMS $406\left(30,[\mathrm{M}]^{+\bullet}\right), 217(100)$; HREIMS $m / z 406.1987[\mathrm{M}]^{+\bullet}$ (calcd for $\left.\mathrm{C}_{22} \mathrm{H}_{30} \mathrm{O}_{7}, 406.1992\right)$.

$\left(2^{\prime} R, 6^{\prime \prime} R\right)$ and $\left(2^{\prime} S, 6^{\prime \prime} R\right)-$ Methyl 2-(2-(6-Acetoxyheptyl)-7-methoxy-4-oxochroman-5-yl)acetate $\left(\left(6^{\prime \prime} R\right)-11\right)$. The title compounds were synthesized in the same manner as $(S)$-11 from $(R)-8:[\alpha]^{20}{ }_{D}$ -23.4 (c 3.60, MeOH). All remaining spectroscopic data were identical to those of $\left(2^{\prime} R, 6^{\prime \prime} S\right)$ and $\left(2^{\prime} S, 6^{\prime \prime} S\right)$-methyl 2-(2-(6acetoxyheptyl)-7-methoxy-4-oxochroman-5-yl)acetate.

(S)-Methyl 2-(2-(6-Acetoxyheptyl)-7-methoxy-4-oxo-4H-chromen-5-yl)acetate ((S)-12). (S)-11 (100 mg, $0.246 \mathrm{mmol})$ was dissolved in dimethylsulfoxide $(1 \mathrm{~mL})$, and the mixture was heated to $90{ }^{\circ} \mathrm{C}$ under an atmosphere of argon. 2-Iodoxybenzoic acid (230 $\mathrm{mg}, 0.810 \mathrm{mmol}$ ) was added, and the solution was stirred until all starting material had been consumed according to TLC $(48 \mathrm{~h})$. The reaction was then cooled to room temperature and diluted with EtOAc $(10 \mathrm{~mL})$. The solution was then washed with saturated aqueous $\mathrm{NaHCO}_{3}$, water, and brine. The organic layer was separated, dried with $\mathrm{MgSO}_{4}$, filtered, and concentrated to give a yellow oil. Purification was achieved via flash column chromatography on silica using 33\% EtOAc in petroleum ether as eluent $\left(R_{f}=0.1\right)$ to give the title compound as a colorless, amorphous solid $(71 \%, 70.6 \mathrm{mg}, 0.175$ mmol): $[\alpha]_{D}^{20}+9.0(c 1.00, \mathrm{MeOH}) ; \mathrm{IR}$ (neat) $\nu_{\max } 1751,1649,1601$ $\mathrm{cm}^{-1} ;{ }^{1} \mathrm{H}$ NMR $\left(300 \mathrm{MHz}, \mathrm{CDCl}_{3}\right) \delta 6.78\left(1 \mathrm{H}, \mathrm{d},{ }^{4} J=2.5 \mathrm{~Hz}, 8^{\prime}-\mathrm{H}\right)$, 
$6.69\left(1 \mathrm{H}, \mathrm{d},{ }^{4} J=2.5 \mathrm{~Hz}, 6^{\prime}-\mathrm{H}\right), 5.99\left(1 \mathrm{H}, \mathrm{s}, 3^{\prime}-\mathrm{H}\right), 4.89\left(1 \mathrm{H}, \mathrm{tq},{ }^{3} J=\right.$ 6.2, $\left.6.2 \mathrm{~Hz}, 6^{\prime \prime}-\mathrm{H}\right), 4.15(2 \mathrm{H}, \mathrm{s}, 2-\mathrm{H}), 3.88\left(3 \mathrm{H}, \mathrm{s}, 7^{\prime}-\mathrm{OCH}_{3}\right), 3.72$ $\left(3 \mathrm{H}, \mathrm{s}, 1-\mathrm{OCH}_{3}\right), 2.53\left(2 \mathrm{H}, \mathrm{t},{ }^{3} \mathrm{~J}=7.7 \mathrm{~Hz}, 1^{\prime \prime}-\mathrm{H}\right), 2.03(3 \mathrm{H}, \mathrm{s}$, $\left.\mathrm{C}(\mathrm{O}) \mathrm{CH}_{3}\right), 1.75-1.25\left(8 \mathrm{H}, \mathrm{m}, 2^{\prime \prime}-5^{\prime \prime}-\mathrm{H}\right), 1.21\left(3 \mathrm{H}, \mathrm{d},{ }^{3} \mathrm{~J}=6.2 \mathrm{~Hz}\right.$, $\left.7^{\prime \prime}-\mathrm{H}\right) ;{ }^{13} \mathrm{C}$ NMR $\left(75 \mathrm{MHz}, \mathrm{CDCl}_{3}\right) \delta 179.1\left(4^{\prime}-\mathrm{C}\right), 171.8(1-\mathrm{C})$, $170.8\left(\mathrm{C}(\mathrm{O}) \mathrm{CH}_{3}\right), 167.5\left(2^{\prime}-\mathrm{C}\right), 162.5\left(7^{\prime}-\mathrm{C}\right), 159.6\left(8^{\prime} \mathrm{a}-\mathrm{C}\right), 137.1$ (5'-C), 117.5 (6'-C), 116.0 (4'a-C), 110.7 (3'-C), 99.9 ( $\left.8^{\prime}-\mathrm{C}\right), 70.8$ $\left(6^{\prime \prime}-\mathrm{C}\right), 55.7\left(7^{\prime}-\mathrm{OCH}_{3}\right), 51.8\left(1-\mathrm{OCH}_{3}\right), 40.9(2-\mathrm{H}), 35.7,33.6,28.8$, 26.6, 25.1, $21.4\left(\mathrm{C}(\mathrm{O}) \mathrm{CH}_{3}\right), 20.0$; ESIMS $427\left(100,[\mathrm{M}+\mathrm{Na}]^{+}\right), 405$ $\left(10,[\mathrm{M}+\mathrm{H}]^{+}\right)$; HRESIMS $\mathrm{m} / z$ 405.1914 $[\mathrm{M}+\mathrm{H}]^{+}$(calcd for $\left.\mathrm{C}_{22} \mathrm{H}_{29} \mathrm{O}_{7}, 405.1913\right)$.

(R)-Methyl 2-(2-(6-Acetoxyheptyl)-7-methoxy-4-oxo-4H-chromen-5-yl)acetate ((R)-12). The title compound was synthesized in the same manner as $(S)$-12 from $(R)-11:[\alpha]^{20}{ }_{\mathrm{D}}-7.1(c 1.20, \mathrm{MeOH})$. All remaining spectroscopic data were identical to those of $(S)$-methyl 2-(2-(6-acetoxyheptyl)-7-methoxy-4-oxo-4H-chromen-5-yl)acetate.

Pestalotiopsone D: (S)-Methyl 2-(7-Hydroxy-2-(6-hydroxyheptyl)4-oxo-4H-chromen-5-yl)acetate ((S)-4). To a solution of (S)-12 (40.0 $\mathrm{mg}, 0.0985 \mathrm{mmol})$ in toluene $(10 \mathrm{~mL})$ was added aluminum chloride $(30.0 \mathrm{mg}, 0.225 \mathrm{mmol})$, and the solution was stirred at reflux for $16 \mathrm{~h}$ under an atmosphere of nitrogen. The reaction was then cooled to room temperature, and ice was added followed by aqueous $1 \mathrm{M} \mathrm{HCl}$. The solution was extracted with EtOAc, and the organic layer was separated, dried with $\mathrm{MgSO}_{4}$, filtered, and concentrated to give a brown oil. Purification via flash column chromatography on silica with $50 \%$ EtOAc in petroleum ether as eluent $\left(R_{f}=0.3\right)$ yielded the title compound as a colorless, amorphous solid (96\%, $32.9 \mathrm{mg}, 0.0946$ $\mathrm{mmol}$ ). Purity was confirmed via HPLC employing a Phenomenex LUX cellulose-4 $5 \mu \mathrm{m}$ enantioselective column $(250 \mathrm{~mm} \times 4.6 \mathrm{~mm})$ using $25 \%$ ethanol in $n$-hexane as solvent, resulting in a retention time of 7.65 min: $[\alpha]^{20}{ }_{D}+12.6(c 0.93, \mathrm{MeOH})$; UV $\left(\mathrm{CH}_{3} \mathrm{OH}\right) \lambda_{\max }(\log \varepsilon)$ 210 (4.19), 242 (4.08), 250 (4.11), 290 (3.91) nm; IR (neat) $\nu_{\max }$ $3492,1712,1600 \mathrm{~cm}^{-1} ;{ }^{1} \mathrm{H}$ NMR $\left(300 \mathrm{MHz}\right.$, acetone- $\left.d_{6}\right) \delta 6.81(1 \mathrm{H}$, d, $\left.{ }^{4} J=2.4 \mathrm{~Hz}, 8^{\prime}-\mathrm{H}\right), 6.74\left(1 \mathrm{H}, \mathrm{d},{ }^{4} \mathrm{~J}=2.4 \mathrm{~Hz}, 6^{\prime}-\mathrm{H}\right), 5.93\left(1 \mathrm{H}, \mathrm{s}, 3^{\prime}-\right.$ $\mathrm{H}), 4.11(2 \mathrm{H}, \mathrm{s}, 2-\mathrm{H}), 3.71\left(1 \mathrm{H}, \mathrm{m}, 6^{\prime \prime}-\mathrm{H}\right), 3.60\left(3 \mathrm{H}, \mathrm{s}, 1-\mathrm{OCH}_{3}\right), 2.59$ $\left(2 \mathrm{H}, \mathrm{t},{ }^{3} J=7.5 \mathrm{~Hz}, 1^{\prime \prime}-\mathrm{H}\right), 1.72\left(2 \mathrm{H}, \mathrm{m}, 2^{\prime \prime}-\mathrm{H}\right), 1.45-1.35(6 \mathrm{H}, \mathrm{m}$, $\left.3^{\prime \prime}-5^{\prime \prime}-\mathrm{H}\right), 1.12\left(3 \mathrm{H}, \mathrm{d},{ }^{3} \mathrm{~J}=6.2 \mathrm{~Hz}, 7^{\prime \prime}-\mathrm{H}\right) ;{ }^{13} \mathrm{C}$ NMR $(125 \mathrm{MHz}$, acetone- $\left.d_{6}\right) \delta 181.0\left(4^{\prime}-\mathrm{C}\right), 173.8(1-\mathrm{C}), 170.5\left(2^{\prime}-\mathrm{C}\right), 165.5$ ( $\left.7^{\prime}-\mathrm{C}\right)$, $162.4\left(8^{\prime} \mathrm{a}-\mathrm{C}\right), 140.4\left(5^{\prime}-\mathrm{C}\right), 120.3\left(6^{\prime}-\mathrm{C}\right), 118.8\left(4^{\prime} \mathrm{a}-\mathrm{C}\right), 113.1\left(3^{\prime}-\right.$ C), $102.6\left(8^{\prime}-\mathrm{C}\right), 69.4\left(6^{\prime \prime}-\mathrm{C}\right), 53.6\left(1-\mathrm{OCH}_{3}\right), 42.9(2-\mathrm{C}), 42.1\left(5^{\prime \prime}\right.$ C), 36.1, 29.5, 28.3, 26.1, $23.5\left(7^{\prime \prime}-\mathrm{C}\right)$; ESIMS $349\left(80,[\mathrm{M}+\mathrm{H}]^{+}\right)$, 334 (100); HRESIMS $m / z 349.1648[\mathrm{M}+\mathrm{H}]^{+}$(calcd for $\mathrm{C}_{19} \mathrm{H}_{25} \mathrm{O}_{6}$, 349.1651).

(R)-Methyl 2-(7-Hydroxy-2-(6-hydroxyheptyl)-4-oxo-4H-chromen-5-yl)acetate ((R)-4). The title compound was synthesized in the same manner as $(S)-4$ from $(R)-12$. Purity was confirmed via HPLC employing a Phenomenex LUX cellulose-4 $5 \mu \mathrm{m}$ enantioselective column $(250 \mathrm{~mm} \times 4.6 \mathrm{~mm})$ using $25 \%$ ethanol in $n$-hexane as solvent, resulting in a retention time of $12.72 \mathrm{~min}:[\alpha]_{\mathrm{D}}^{20}-10.8(c$ $0.81, \mathrm{MeOH})$. All remaining spectroscopic data were identical to those of (S)-methyl 2-(7-hydroxy-2-(6-hydroxyheptyl)-4-oxo-4H-chromen5-yl)acetate.

Pestalotiopsone E: (S)-Ethyl 2-(7-Hydroxy-2-(6-hydroxyheptyl)-4oxo-4H-chromen-5-yl)acetate ((S)-5). To a solution of $(S)-4$ (10.0 $\mathrm{mg}, 28.6 \mu \mathrm{mol})$ in ethanol $(5 \mathrm{~mL})$ was added 2 drops of sulfuric acid. The reaction was stirred at $50{ }^{\circ} \mathrm{C}$ for $24 \mathrm{~h}$, at which time water was added and the solution was extracted with ethyl acetate. The combined organic layers were washed with aqueous $\mathrm{NaHCO}_{3}$, dried with $\mathrm{MgSO}_{4}$, filtered, and concentrated to give the title compound as a colorless, amorphous solid (99\%, $10.3 \mathrm{mg}, 28.4 \mu \mathrm{mol})$. Purity was confirmed via HPLC employing a Phenomenex LUX cellulose-4 $5 \mu \mathrm{m}$ enantioselective column $(250 \mathrm{~mm} \times 4.6 \mathrm{~mm})$ using $25 \%$ ethanol in $n$ hexane as solvent, resulting in a retention time of $15.91 \mathrm{~min}:[\alpha]^{20}{ }_{\mathrm{D}}$ +14.1 (c 0.73, MeOH); UV (EtOH) $\lambda_{\max }(\log \varepsilon) 208$ (4.19), 242 (4.16), 250 (4.20), $292(4.03) \mathrm{nm}$; IR (neat) $\nu_{\max } 3339,1617,1599$ $\mathrm{cm}^{-1} ;{ }^{1} \mathrm{H}$ NMR $\left(300 \mathrm{MHz}\right.$, acetone- $\left.d_{6}\right) \delta 6.80\left(1 \mathrm{H}, \mathrm{d},{ }^{4} J=2.3 \mathrm{~Hz}, 8^{\prime}\right.$ $\mathrm{H}), 6.74\left(1 \mathrm{H}, \mathrm{d},{ }^{4} J=2.3 \mathrm{~Hz}, 6^{\prime}-\mathrm{H}\right), 5.93\left(1 \mathrm{H}, \mathrm{s}, 3^{\prime}-\mathrm{H}\right), 4.10(2 \mathrm{H}, \mathrm{s}, 2-$ $\mathrm{H}), 4.07\left(2 \mathrm{H}, \mathrm{q},{ }^{3} \mathrm{~J}=7.1 \mathrm{~Hz}, 1-\mathrm{OCH}_{2} \mathrm{CH}_{3}\right), 3.70\left(1 \mathrm{H}, \mathrm{m}, 6^{\prime \prime}-\mathrm{H}\right), 2.59$ $\left(2 \mathrm{H}, \mathrm{t},{ }^{3} \mathrm{~J}=7.4 \mathrm{~Hz}, 1^{\prime \prime}-\mathrm{H}\right), 1.72\left(2 \mathrm{H}, \mathrm{m}, 2^{\prime \prime}-\mathrm{H}\right), 1.45-1.35\left(6 \mathrm{H}, \mathrm{m}, 3^{\prime \prime}-\right.$ $\left.5^{\prime \prime}-\mathrm{H}\right), 1.20\left(3 \mathrm{H}, \mathrm{t},{ }^{3} \mathrm{~J}=7.1 \mathrm{~Hz}, 1-\mathrm{OCH}_{2} \mathrm{CH}_{3}\right), 1.12\left(3 \mathrm{H}, \mathrm{d},{ }^{3} \mathrm{~J}=6.2\right.$
$\left.\mathrm{Hz}, 7^{\prime \prime}-\mathrm{H}\right) ;{ }^{13} \mathrm{C}$ NMR (125 MHz, acetone- $\left.d_{6}\right) \delta 181.1$ (4'-C), 174.0 (1-C), 170.6 (2'-C), 165.7 (7'-C), 162.7 ( $\left.8^{\prime} \mathrm{a}-\mathrm{C}\right), 140.3$ (5'-C), 120.3 (6'-C), 118.7 (4'a-C), 113.4 (3'-C), $102.6\left(8^{\prime}-\mathrm{C}\right), 69.4\left(6^{\prime \prime}-\mathrm{C}\right), 61.0$ (1- $\left.\mathrm{OCH}_{2} \mathrm{CH}_{3}\right), 42.9$ (2-C), 42.1 (5"-C), 36.1, 29.6, 28.3, 26.1, 23.5 (7"-C), $14.5\left(1-\mathrm{OCH}_{2} \mathrm{CH}_{3}\right)$; ESIMS $363\left(20,[\mathrm{M}+\mathrm{H}]^{+}\right), 334(100)$; HRESIMS $m / z 363.1804[\mathrm{M}+\mathrm{H}]^{+}$(calcd for $\mathrm{C}_{20} \mathrm{H}_{27} \mathrm{O}_{6}, 363.1808$ ).

(R)-Ethyl 2-(7-Hydroxy-2-(6-hydroxyheptyl)-4-oxo-4H-chromen5 -yl)acetate $((R)-5)$. The title compound was synthesized in the same manner as $(S)-5$ from $(R)-4$. Purity was confirmed via HPLC employing a Phenomenex LUX cellulose-4 $5 \mu \mathrm{m}$ enantioselective column $(250 \mathrm{~mm} \times 4.6 \mathrm{~mm})$ using $25 \%$ ethanol in $n$-hexane as solvent, resulting in a retention time of $18.07 \mathrm{~min}:[\alpha]^{20}{ }_{\mathrm{D}}-15.8(c$ $0.62, \mathrm{MeOH})$. All remaining spectroscopic data were identical to those of (S)-ethyl 2-(7-hydroxy-2-(6-hydroxyheptyl)-4-oxo-4H-chromen-5yl)acetate.

Determination of the Sensitivity of Selected Bacterial Strains against Selected Compounds. The sensitivity of four clinical bacterial strains (Staphylococcus epidermidis, Esherichia coli, Pseudomonas aeruginosa, and Mycobacterium smegmatis), was tested by a turbidity assay $\left(\mathrm{OD}_{600}\right.$, optical density at $\left.\lambda=600 \mathrm{~nm}\right){ }^{7}$ To prepare the inoculum, the bacterial suspension adjusted to equal the density of $0.5 \mathrm{McF}$ arland standard $\left(\mathrm{OD}_{600}=0.08\right)$ was diluted 1:100 with Mueller Hinton broth (MHB), and $150 \mu \mathrm{L}$ was used as an inoculum. Then $40 \mu \mathrm{L}$ of MHB was added to wells $1 \mathrm{~B}$ to $1 \mathrm{H}$ of sterile $96-$ well plates. A $80 \mu \mathrm{L}$ portion of the unfiltered test sample $(3 \mathrm{mg} / \mathrm{mL})$ or appropriate antibiotic control $(1 \mathrm{mg} / \mathrm{mL})$ was dispensed into well $1 \mathrm{~A}$, $40 \mu \mathrm{L}$ was removed, and serial 2 -fold dilutions of the test sample were prepared directly on the plate. The plate was read at $A_{600}$ to control for pre-existing turbidity of the samples before incubation. The plate was incubated at $37^{\circ} \mathrm{C}$ for $18 \mathrm{~h}$ on a shaker incubator. After incubation, the plate was read at $A_{600}$ to assess the relative turbidity (i.e., growth) of the treated cultures; 50\% inhibition was determined based on a comparison with the average turbidity readings of the untreated control. Inhibition was calculated as

$$
\mathrm{IC}_{50}=10[\log (A / B) \times(50-C) /(D-C)+\log (B)]
$$

where $A$ is the higher concentration of test compound of the two points on the graph that bracket $50 \%$ inhibition, $B$ is the lower concentration of test compound of the two points on the graph that bracket $50 \%$ inhibition, $C$ is the inhibitory activity (\%) at the concentration $B$, and $D$ is the inhibitory activity (\%) at the concentration $A$.

\section{ASSOCIATED CONTENT}

\section{S Supporting Information}

${ }^{1} \mathrm{H}$ and ${ }^{13} \mathrm{C}$ NMR spectra for all synthetic compounds and analytical data for the synthesized natural products. This material is available free of charge via the Internet at http:// pubs.acs.org.

\section{AUTHOR INFORMATION}

\section{Corresponding Author}

*E-mail: rab@anu.edu.au. Tel: +61 261253419.

\section{Author Contributions}

The manuscript was written through contributions of all authors. All authors have given approval to the final version of the manuscript.

\section{Notes}

The authors declare no competing financial interest.

\section{ACKNOWLEDGMENTS}

This study was undertaken with the support of the ANU and an NHMRC project grant (1028092). A.M.B. acknowledges the ANU for an Australian Postgraduate Award. 


\section{REFERENCES}

(1) (a) Kim, H. W.; Kim, B. K. Int. J. Med. Mushrooms 1999, 1, 121138. (b) Shiao, M.-S. Chem. Rec. 2003, 3, 172-180. (c) Paterson, R. R. M. Phytochemistry 2008, 69, 1469-1495.

(2) (a) Tejesvi, M. V.; Mahesh, B.; Nalini, M. S.; Prakash, H. S.; Kini, K. R.; Subbiah, V.; Shetty, H. S. Can. J. Microbiol. 2006, 52, 427-435.

(b) Bhagobaty, R. K.; Joshi, S. R. Pharmacologyonline 2008, 882-888.

(c) Bhagobaty, R. K.; Joshi, S. R.; Kumar, R. Afr. J. Microbiol. Res. 2010, 4, 766-770. (d) Bhagobaty, R. K.; Joshi, S. R. J. Metabolomics Syst. Biol. 2011, 2, 20-31. (e) Bhagobaty, R. K.; Joshi, S. R. Biotechnol. Bioprocess Eng. 2012, 17, 33-40. (f) Nath, A.; Raghunatha, P.; Joshi, S. R. Mycobiology 2012, 40, 8-13.

(3) Xu, J.; Kjer, J.; Sendker, J.; Wray, V.; Guan, H.; Edrada, R.; Lin, W.; Wu, J.; Proksch, P. J. Nat. Prod. 2009, 72, 662-665.

(4) (a) Machado, N. F. L.; Marques, M. P. M. Curr. Bioact. Compd. 2010, 6, 76-89. (b) Sharma, S. K.; Kumar, S.; Chand, K.; Kathuria, A.;

Gupta, A.; Jain, R. Curr. Med. Chem. 2011, 18, 3825-3852.

(c) Khadem, S.; Marles, R. J. Molecules 2012, 17, 191-206.

(5) Beekman, A. M.; Castillo Martinez, E.; Barrow, R. A. Org. Biomol. Chem. 2013, 11, 1109-1115.

(6) Nicolaou, K. C.; Montagnon, T.; Baran, P. S.; Zhong, Y. L. J. Am. Chem. Soc. 2002, 124, 2245-2258.

(7) (a) Chand, S.; Lusunzi, I.; Veal, D.; Williams, L.; Karuso, P. J. Antibiot. (Tokyo) 1994, 47, 1295-1304. (b) Steenkamp, V.; Mathivha, E.; Gouws, M. C.; van Rensburg, C. E. J. J. Ethnopharmacol. 2004, 95, 353-357. 